\title{
Lipids and Atherogenic Indices Fluctuation in Rheumatoid Arthritis Patients on Long-Term Tocilizumab Treatment
}

\author{
Fabio Cacciapaglia (D), Maria Grazia Anelli, Angela Rinaldi, Marco Fornaro, \\ Giuseppe Lopalco $(\mathbb{D}$, Crescenzio Scioscia, Giovanni Lapadula, and Florenzo Iannone
}

\author{
Rheumatology Unit-Department of Emergence Medicine and Transplantation (DETO), University of Bari "Aldo Moro", \\ 70124 Bari, Italy \\ Correspondence should be addressed to Fabio Cacciapaglia; fabio.cacciapaglia@policlinico.ba.it
}

Received 5 May 2018; Revised 7 September 2018; Accepted 19 September 2018; Published 14 October 2018

Academic Editor: Kutty Selva Nandakumar

Copyright (C) 2018 Fabio Cacciapaglia et al. This is an open access article distributed under the Creative Commons Attribution License, which permits unrestricted use, distribution, and reproduction in any medium, provided the original work is properly cited.

\begin{abstract}
Rheumatoid arthritis (RA) patients are at high risk of cardiovascular (CV) events, and the chronic inflammatory state may generate quantitative and qualitative changes in lipoprotein fractions. The anti-IL-6 receptor tocilizumab (TCZ), even if effective in inflammation and joint damage prevention, determined significant alterations to RA patients' lipid levels in randomized controlled trials, but real-world data are lacking. We evaluated the changes in lipid fraction levels and disease activity in a longitudinal cohort of RA patients on long-term treatment with tocilizumab (TCZ) in a community setting. We retrospectively selected 40 naïve-biologic RA patients on treatment with intravenous TCZ compared to 20 RA patients on methotrexate treatment as the control group. Total cholesterol (Tot-Chol), low-density lipoproteins (LDL), high-density lipoprotein (HDL), and triglyceride (TG) levels were measured at the baseline and at 12, 24, and 52 weeks thereafter. At the same points, 28-joint disease activity score (DAS28), clinical disease activity index (CDAI), and EULAR clinical responses were also assessed. During the first 24 weeks, we observed in TCZ-treated patients a progressive statistically significant $(p<0.001)$ increase in Tot-Chol, LDL, HDL, and TG, which returned close to the baseline at 52 weeks. But no changes in the lipid-related CV risk indices Tot-Chol/HDL and LDL/HDL ratios and the atherogenic index $\left(\log _{10}\right.$ TG/HDL) were detectable. Notably, we observed a statistically significant negative correlation between changes in lipid fractions and DAS28 or CDAI. The prolonged treatment with TCZ was associated to a transient increase in cholesterol's fractions during the first 6 months of treatment, with inverse correlation to disease activity, but with no impact on surrogate lipid indices of atherogenic risk. These findings may aid clinicians in interpreting the RA patient's lipid profile in daily clinical practice.
\end{abstract}

\section{Introduction}

Patients with rheumatoid arthritis (RA) have a twofold increased risk of morbidity and mortality for cardiovascular $(\mathrm{CV})$ disease $[1,2]$, and "traditional" CV risk factors such as high blood pressure, diabetes, smoking, and dyslipidemia are not enough to justify this risk [3-6]. According to recent evidence, the state of low-grade systemic inflammation seems to be a key element in determining a condition of "accelerated" atherosclerosis [7, 8]. Moreover, the chronic inflammation seems to be able to interfere with traditional CV risk factors such as lipoprotein metabolism, leading to quantitative and qualitative changes in triglycerides and low-density lipoprotein (LDL) and high-density lipoprotein (HDL) cholesterol fractions $[5,9,10]$. Indeed, a condition of "reverse epidemiology" has been also taken into account for RA patients [11]. RA patients with active disease, despite the low levels of total and LDL cholesterol, have increased $\mathrm{CV}$ risk that subsides in those patients in clinical disease remission but with a lipid profile otherwise considered "proatherogenic," thus leading to a "lipid paradox" $[5,11]$. Several studies showed a reduction in CV risk during effective control of disease activity both under synthetic and biological disease-modifying antirheumatic drugs (DMARDs) [12-15]. The antagonism of interleukin-6 (IL-6) with tocilizumab (TCZ), an IL-6 receptor antagonist, has been 
associated in randomized clinical trials with changes in the lipoprotein profile, with reports of significant increase in total and LDL cholesterol levels after 8-12 weeks of treatment $[16,17]$. These findings can raise concern in clinical $\mathrm{CV}$ risk management of RA patients treated with TCZ [18], although mechanisms and long-term outcomes are still unclear [19-21]. Therefore, the aim of this study was to evaluate in a community setting the effect of one-year TCZ treatment on RA patient's lipid metabolism.

\section{Materials and Methods}

Forty patients with diagnosis of RA, according to the 2010 EULAR/ACR classification criteria [22], with an inadequate response to previous synthetic DMARD treatment and naïve to biologic agents, which had started combination treatment with intravenous TCZ ( $8 \mathrm{mg} / \mathrm{kg}$ every 28 days) in our outpatient clinic from the $1^{\text {st }}$ January until the $31^{\text {st }}$ December 2015, were retrospectively evaluated. Twenty RA patients treated for at least 52 weeks with methotrexate (MTX) were considered as the control group. Demographics of enrolled patients are reported in Table 1. According to daily clinical practice, all patients have been screened for latent TB infection, exposure to $\mathrm{B}$ and $\mathrm{C}$ hepatitis, HIV infection, and anamnestic exposure to the varicella zoster virus and evaluated for previous tumors. Subjects treated with steroid $>7.5 \mathrm{mg}$ /day prednisone equivalent, lipid-lowering agents, and/or smoking more than 10 cigarettes per day were excluded from the study. The functional capacity of patients was evaluated with the Health Assessment Questionnaire Disability Index (HAQ-DI).

At the baseline and after 12,24, and 52 weeks of treatment, the DAS28-ESR and CDAI were saved, and the treatment response according to the EULAR definitions [23] was assessed. At the same time, plasma levels of total cholesterol (Tot-Chol), low-density lipoproteins (LDL), high-density lipoprotein (HDL), and triglycerides (TG), added to the routine rheumatology laboratory tests and assessed according to standard laboratory protocol, were retrieved. The non-HDLcholesterol (Tot-Chol-HDL) levels, the lipid-related CV risk indices Tot-Chol/HDL and LDL/HDL ratios, and the atherogenic index of plasma (AIP: $\log _{10}$ TG/HDL) were also calculated [24-26].

As part of the GISEA registry protocol approved by the local Ethical Committee (Trial.Gov NCT01543594), all patients had signed a written informed consent before starting the treatment, and this observational study was conforming to the ethical guidelines of the 1975 Declaration of Helsinki.

2.1. Statistical Analysis. All data have been tested for the D'Agostino-Pearson omnibus normality test, and Gaussian distribution was detected; accordingly, we expressed data as mean \pm standard deviation (SD) or percentage with confidence interval when appropriate. Consequently, differences for continuous variables were evaluated using the two-tailed variable analysis of variance followed by paired $t$-test, while categorical data were calculated using Fisher's probability test or the $\chi^{2}$-test, when appropriate. To test whether the difference in means between the groups was
TABlE 1: Baseline demographic characteristics of RA patients treated with tocilizumab (TCZ) and methotrexate (MTX).

\begin{tabular}{lcc}
\hline & $\begin{array}{c}\text { TCZ pts. } \\
(n=40)\end{array}$ & $\begin{array}{c}\text { MTX pts. } \\
(n=20)\end{array}$ \\
\hline Female, $n(\%)$ & $36(90)$ & $12(60)^{*}$ \\
Age (years)_mean \pm SD & $55 \pm 11$ & $56 \pm 14$ \\
Disease duration (months)_mean \pm SD & $9.8 \pm 4.3$ & $6.7 \pm 3.0^{*}$ \\
BMI $\left(\mathrm{kg} / \mathrm{m}^{2}\right)$-mean \pm SD & $26.5 \pm 4.2$ & $27.2 \pm 5.0$ \\
Smokers, $n(\%)$ & $11(27.5)$ & $5(25)$ \\
Arterial hypertension, $n(\%)$ & $12(30)$ & $7(35)$ \\
Diabetes, $n(\%)$ & $6(15)$ & $3(15)$ \\
RF positive, $n(\%)$ & $32(80)$ & $16(80)$ \\
ACPA positive, $n(\%)$ & $33(82.5)$ & $17(85)$ \\
Combination treatment, $n(\%)$ & & \\
$\quad$ Steroids & $14(35)$ & $6(30)$ \\
csDMARD & & \\
$\quad$ MTX & $28(70)$ & $20(100)$ \\
LFM & $8(20)$ & \\
SSZ & $4(10)$ & \\
\hline ACPA
\end{tabular}

ACPA: anticitrullinated peptide antibody; csDMARD: conventional synthetic disease-modifying antirheumatic drug; MTX: methotrexate; LFM: leflunomide; SSZ: sulfasalazine; RF: rheumatoid factor. ${ }^{*} p<0.01$.

statistically significant, we performed the analysis of variance (ANOVA). Correlations between continuous variables were performed using the Pearson test.

The analysis of covariance (ANCOVA) was used to determine confounding effect as well as to expose eventual interaction effect of suitable variables on the relationship between lipid changes at 24 and 52 weeks of treatment with TCZ or MTX. Parameters tested were disease duration, gender, baseline mean ESR and CRP serum levels, mean lipid levels and fractions, baseline mean DAS28, mean CDAI, and mean HAQ. Confounders were fitted in the adjusted model along with TCZ treatment as the independent variable.

The calculated statistical power of the study, considering an alpha error level of $5 \%$ with a sample size of 40 patients and a nominal variation of $10 \pm 5 \mathrm{mg} / \mathrm{dl}$ in lipid levels, was approximately $99 \%$.

A $p$ value $<0.05$ or $<0.2$ was considered statistically significant in the univariate or multivariate model, as appropriate. The Statistical System Prism (GraphPad InStat, version 6.0-GraphPad Software, San Diego, CA, USA) and the Analysis System (SAS 9.3 Software-SAS Institute Inc., Cary, NC, USA) were used for all analysis.

\section{Results}

At baseline, all patients had high disease activity (DAS28ESR > 5.1 with CDAI > 22), and, as shown in Table 1, a significant improvement of both parameters DAS28 and CDAI was achieved as early as 24 weeks and maintained until 52 weeks of treatment $(p<0.001)$. After 12 weeks of TCZ, 16 patients had an improvement in DAS28 $\geq 1.2$ achieving a 
TABLE 2: Laboratory and clinical findings of RA patients treated with tocilizumab (TCZ) and methotrexate (MTX).

\begin{tabular}{|c|c|c|c|c|c|c|}
\hline \multirow[b]{2}{*}{$($ Mean \pm SD $)$} & \multicolumn{3}{|c|}{ TCZ pts. $(n=40)$} & \multicolumn{3}{|c|}{ MTX pts. $(n=20)$} \\
\hline & Baseline & 24 weeks & 52 weeks & Baseline & 24 weeks & 52 weeks \\
\hline $\operatorname{ESR}(\mathrm{mm} / \mathrm{h})$ & $64 \pm 23$ & $17 \pm 9^{*}$ & $15 \pm 7^{*}$ & $57 \pm 23$ & $13 \pm 12^{*}$ & $12 \pm 10^{*}$ \\
\hline CRP (mg/l) & $7.3 \pm 3.2$ & $1.1 \pm 3^{*}$ & $0.9 \pm 2.7^{*}$ & $6.8 \pm 1.9$ & $3.5 \pm 4^{*} \S$ & $1.7 \pm 1^{*} \S$ \\
\hline DAS28 & $5.3 \pm 0.4$ & $2.5 \pm 1.6^{*}$ & $2.2 \pm 1.1^{*}$ & $4.9 \pm 1.5$ & $2.6 \pm 1.8^{*}$ & $1.9 \pm 0.8^{*}$ \\
\hline CDAI & $28.9 \pm 2.1$ & $6.1 \pm 1.1^{*}$ & $4.7 \pm 1^{*}$ & $26.6 \pm 8.6$ & $9 \pm 1.4^{*}$ & $5.2 \pm 1.1^{*}$ \\
\hline HAQ & $1.7 \pm 0.5$ & $0.8 \pm 0.4^{*}$ & $0.7 \pm 0.5^{*}$ & $1.2 \pm 0.7 \S$ & $0.4 \pm 0.3^{*} \S$ & $0.2 \pm 0.5^{*} \S$ \\
\hline Total cholesterol (mg/dl) & $210 \pm 21$ & $235 \pm 23^{*} \dagger$ & $223 \pm 24^{*}$ & $189 \pm 38$ & $188 \pm 35 \S$ & $192 \pm 38 \$$ \\
\hline Non-HDL cholesterol (mg/dl) & $166 \pm 18$ & $187 \pm 16^{*} \dagger$ & $172 \pm 14$ & $156 \pm 27$ & $146 \pm 25 \S$ & $142 \pm 19 \S$ \\
\hline LDL cholesterol (mg/dl) & $146 \pm 13$ & $161 \pm 11^{*} \dagger$ & $151 \pm 12$ & $128 \pm 22 \S$ & $117 \pm 25 \S$ & $118 \pm 15 \S$ \\
\hline Triglycerides (mg/dl) & $139 \pm 14$ & $157 \pm 15^{*} \dagger$ & $148 \pm 14^{*}$ & $126 \pm 26 \$$ & $121 \pm 24 \S$ & $123 \pm 22 \S$ \\
\hline HDL cholesterol (mg/dl) & $43 \pm 11$ & $52 \pm 11^{*} \dagger$ & $45 \pm 12$ & $50 \pm 14$ & $48 \pm 12$ & $51 \pm 10$ \\
\hline Total/HDL cholesterol ratio & $4.9 \pm 0.22$ & $4.75 \pm 0.23$ & $4.75 \pm 0.18$ & $3.6 \pm 0.9 \S$ & $3.8 \pm 0.8 \$$ & $3.5 \pm 0.7 \S$ \\
\hline LDL/HDL cholesterol ratio & $3.22 \pm 0.17$ & $3.25 \pm 0.22$ & $3.21 \pm 0.2$ & $2.2 \pm 0.6 \$$ & $2.5 \pm 0.9 \S$ & $2.1 \pm 0.5 \S$ \\
\hline AIP $\left(\log _{10}\right.$ TG/HDL $)$ & $0.51 \pm 0.3$ & $0.48 \pm 0.4$ & $0.52 \pm 0.4$ & $0.4 \pm 0.3 \S$ & $0.4 \pm 0.2 \S$ & $0.38 \pm 0.4 \S$ \\
\hline
\end{tabular}

AIP: atherogenic index of plasma; CRP: C-reactive protein; ESR: erytro-sedimentation rate; DAS28: 28-joint disease activity score; CDAI: clinical disease activity index; HAQ: Health Assessment Questionnaire; HDL: high-density lipoprotein; LDL: low-density lipoprotein; TG: triglycerides. Univariate analysis: ${ }^{*} p<0.001$ vs. baseline; $\$ p<0.01$ vs. TCZ. Multivariate analysis: $\dagger p=0.01$.

good EULAR response, 22 patients had a moderate response DAS28 improvement between 1.2 and 0.6 , and 2 patients had no response (DAS28 improvement $<0.6$ ), according to the treatment response EULAR definitions. Due to failure in clinical response, these last 2 patients dropped out of the study and were no further considered for the statistical analysis. After 24 weeks, further 8 patients achieved a good EULAR response, and at 52 weeks, a total of 33 patients $(82.5 \%)$ had a good clinical response. The HAQ-DI of patients improved significantly from $1.7 \pm 0.5$ of the baseline to $1.2 \pm 0.6,0.8 \pm 0.4$, and $0.7 \pm 0.5$ after 12,24 , and 52 weeks of treatment, respectively $(p<0.001)$.

Methotrexate-treated patients were fewer female and presented lower disease duration compared to TCZ patients but had significant good response to treatment and continued MTX for 52 weeks as due by inclusion criteria.

From the lipid level evaluation of enrolled patients, we detected that in the TCZ group, hypercholesterolemia (TotChol $>200 \mathrm{mg} / \mathrm{dl}$ ) and/or hyper-LDL-Chol levels $(>130 \mathrm{mg} /$ $\mathrm{dl})$ at the baseline was detectable in $32(80 \%)$ of the enrolled patients. Only 8 patients (20\%) had reduced HDL-Chol levels $(<40 \mathrm{mg} / \mathrm{dl})$ and/or hyper-triglyceridemia $(\mathrm{TG}>150 \mathrm{mg} / \mathrm{dl})$. While in MTX-treated patients, only 3 patients (15\%) had a condition of hypercholesterolemia and/or hyper-LDL-Chol levels. In Table 2 are reported the lipid fraction levels at the baseline and after 24 and 52 weeks of treatment with TCZ and MTX. After starting TCZ treatment, Tot-Chol levels had a statistically significant change already after 12 weeks with an average increase of $27 \pm 13 \mathrm{mg} / \mathrm{dl}$ that reached a mean increase of $31 \pm 16 \mathrm{mg} / \mathrm{dl}$ and $12 \pm 15 \mathrm{mg} / \mathrm{dl}$ at 24 weeks and 52 weeks of treatment, respectively, compared to baseline levels $(p<0.05)$ (Figure 1$)$. A similar statistically significant increase was also detected for the HDL- and LDL-Chol and TG levels, with an average increase of $12 \pm 15 \mathrm{mg} / \mathrm{dl}$, $19 \pm 23 \mathrm{mg} / \mathrm{dl}$, and $21 \pm 29 \mathrm{mg} / \mathrm{dl}$ after 12 weeks, respectively, which persisted until week 24 , and settle down to a mean increase of $8 \pm 15 \mathrm{mg} / \mathrm{dl}$ for HDL-Chol, $6 \pm 17 \mathrm{mg} / \mathrm{dl}$ for LDL-Chol, and $8 \pm 24 \mathrm{mg} / \mathrm{dl}$ for TG after 52 weeks compared to baseline levels. From the evaluation of non-HDL-Chol, it was $172 \pm 17 \mathrm{mg} / \mathrm{dl}$ at the baseline and increased to $191 \pm 20 \mathrm{mg} / \mathrm{dl}$ and $192 \pm 20 \mathrm{mg} / \mathrm{dl}$, respectively, after 12 and 24 weeks of treatment ( $p<0.001$ vs. baseline). After 52 weeks of TCZ treatment, it returned to $179 \pm 15 \mathrm{mg} / \mathrm{dl}$ ( $p=0.07$ vs. baseline; $p<0.01$ vs. 12 and 24 weeks). Accordingly, as shown in Figure 2, the ratios Tot-Chol/HDL-Chol and LDL-Chol/HDL-Chol and the AIP remained unchanged during the period of the study, with values considered of "moderate risk," according to the Framingham study [26-29].

In the MTX-treated patients, the lipid levels did not change significantly during treatment but were significantly lower than those observed in TCZ-treated patients (see Table 2). Similarly, the lipid ratios Tot-Chol/HDL-Chol and LDL-Chol/HDL-Chol and the AIP remained unchanged during MTX treatment but were significantly lower than those calculated in TCZ-treated patients.

From the one-way ANCOVA, we observed statistically significant differences in lipid levels after 24 and 52 weeks of treatment among TCZ and MTX arms. However, only baseline mean total cholesterol was found to confound this association, and no interaction effect was found. After adjusting for baseline variables (disease duration, gender, baseline mean ESR and CRP serum levels, baseline mean Tot-Chol, LDL- and HDL- cholesterol and TG levels, baseline mean DAS28 and mean CDAI, and mean HAQ), difference between lipid levels determined at 24 weeks among the TCZ and MTX arms persisted $(p=0.01)$, while statistical significance after 52 weeks of treatment was lost $(p=0.31)$.

Correlating the Tot-Chol, LDL- and HDL- cholesterol, and TG plasmatic level changes, with the DAS28 and CDAI changes after 24 weeks of tocilizumab (TCZ) compared to 


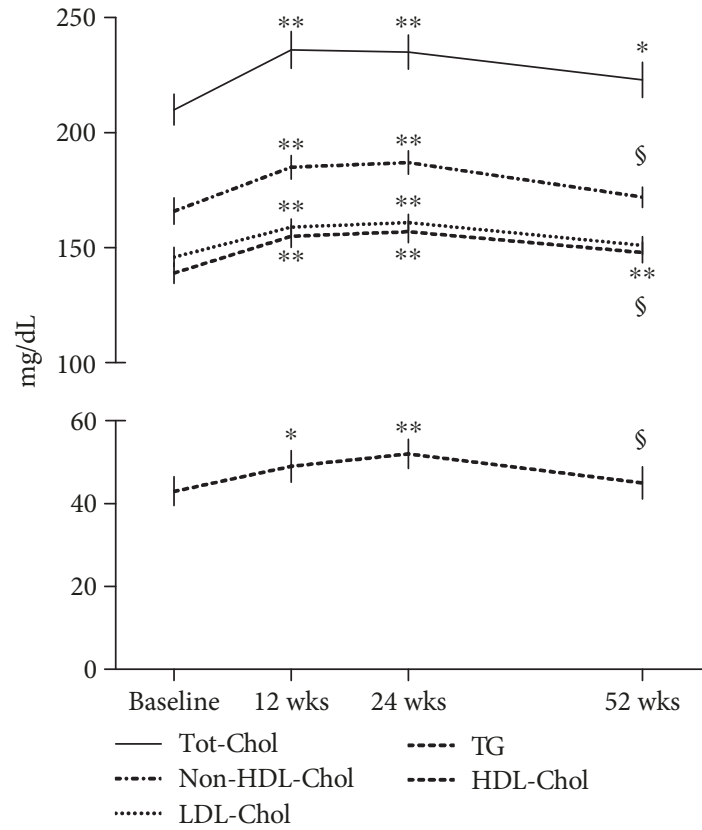

FIgure 1: Changes in total cholesterol (Tot-Chol), non-HDL cholesterol, low-density lipoprotein (LDL) and high-density lipoprotein (HDL) cholesterol, and triglyceride (TG) plasmatic levels during tocilizumab treatment. The peak change in Tot-Chol is at 12 weeks and in non-HDL-, LDL-, and HDL-Chol, and TG is at 24 weeks; after that, lipids come down by 52 weeks but remain still significantly elevated compared to the baseline only for TotChol and TG. ${ }^{*} p<0.05$ and ${ }^{* *} p<0.001$ vs. baseline; $\$ p<0.05$ vs. 24 weeks.

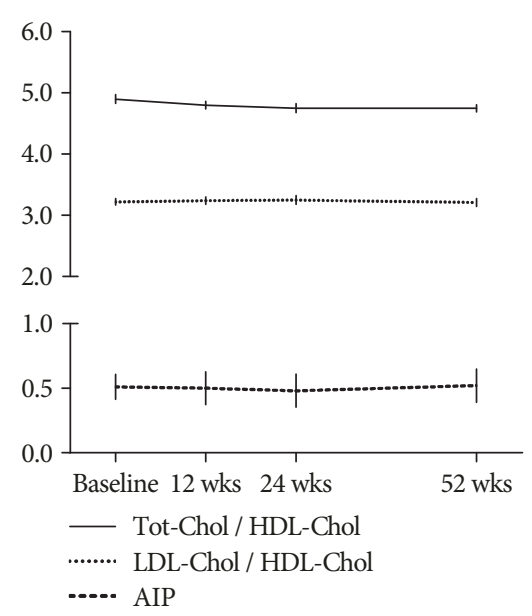

FIgURE 2: Ratios of total cholesterol/high-density lipoprotein (TotChol/HDL-Chol) and low-density lipoprotein-/HDL-cholesterol (LDL-Chol/HDL-Chol) and the atherogenic index of plasma (log triglycerides/HDL-Chol; AIP) at the different time points during tocilizumab treatment. $p=$ NS.

the baseline, we found a statistically significant inverse correlation between lipid levels and disease activity assessed by both scores (Figure 3). Finally, during the 1-year follow-up, there were no $\mathrm{CV}$ events in the studied population.

\section{Discussion}

In our observational study in a real-life setting, we confirmed that during TCZ therapy, all lipid fractions increase, but the prolonged follow-up for 12 months revealed that the increase occurs during the first 6 months of treatment, with a trend to return to baseline levels after one year.

MTX-treated patients showed no significant change in lipid fraction levels over time, confirming the key role of IL-6 in lipid metabolism. It must be highlighted that the control group with MTX had lower basal LDL cholesterol and TG levels, but this difference may be justified by different disease durations and previous csDMARDs \pm steroid treatment in TCZ-treated patients.

Indeed, IL-6 exerts specific effect on hepatic protein production, such as CPR and lipoproteins, and its inhibition with TCZ had more consistent impact on CPR and lipid fractions compared to MTX-treated patients.

Previous studies with TCZ had shown an increase of TotChol levels and LDL and HDL fractions [16, 17], but other studies did not show statistically significant variations [30]. However, all this information comes from randomized clinical trials and their post hoc analysis, with a follow-up of at most 24 weeks. Real-world data are missing, and only the short-term changes on lipid fractions, probably influenced by the anti-inflammatory and hepatic effects on lipid metabolism by the inhibition of IL-6, have been documented.

Hashizume et al. [31] have shown that IL-6 decreases levels of blood lipids, increasing the expression of the LDL receptor in different tissues. Moreover, Strang et al. demonstrated a significant decreased LDL receptor expression in liver cell cultures following TCZ incubation [16]. A recent study by Robertson et al. highlighted that TCZ was able to reduce LDL hypercatabolism in 11 RA patients with high disease activity, leading to Tot-Chol, LDL-Chol, and HDL-Chol elevations, without change in the Tot-Chol/HDL-Chol ratio [21]. Another study [32] reported a reduction of the secretory phospholipase A2 (sPLA2-IIA) and serum amyloid A (SAA), during therapy with TCZ, with modification in HDL lipoprotein composition. More recently, García-Gómez et al. on behalf of the CARdiovascular rheuMAtology study project reported from their database that the $30 \mathrm{RA}$ patients treated with TCZ had statistically significant lower Lp(a) plasma levels compared to patients treated with nonbiologic therapies [33].

Such effects result in a potential antiatherogenic and anti-inflammatory lipoprotein's function, restoring their scavenger function to the liver. In this way, the IL- 6 antagonism would result in good inflammation control despite an increase in serum lipid levels, with a favorable net effect on $\mathrm{CV}$ risk. The systemic inflammatory condition observed in high-disease-activity RA patients and the high IL-6 levels increases the cholesterol uptake from the liver and other tissues by the overexpression of LDL receptors $[16,31,34]$. The reduction of IL-6 levels, such as in low-disease or remission RA patients, even more during an anti-IL-6 treatment, leads to a reduction in the reuptake of cholesterol fractions from tissues with consequent increase in their circulating levels. Finally, the good disease and systemic inflammation control, obtained with prolonged effective anti-IL-6 treatment, would 


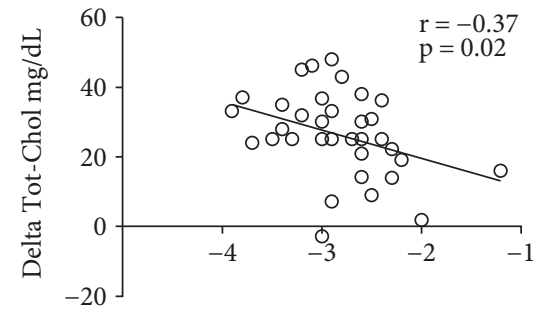

Delta DAS28
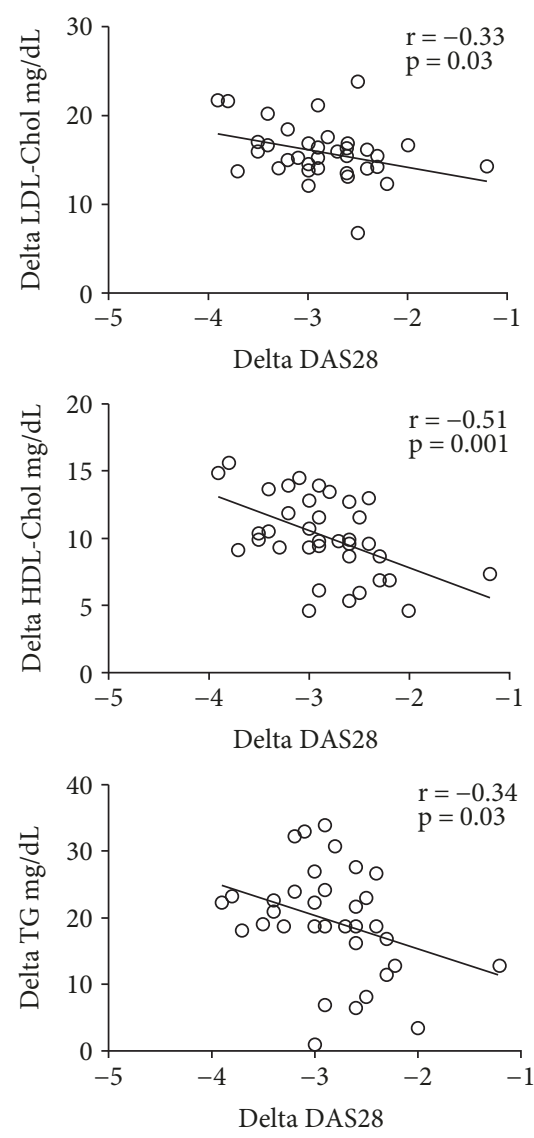

(a)

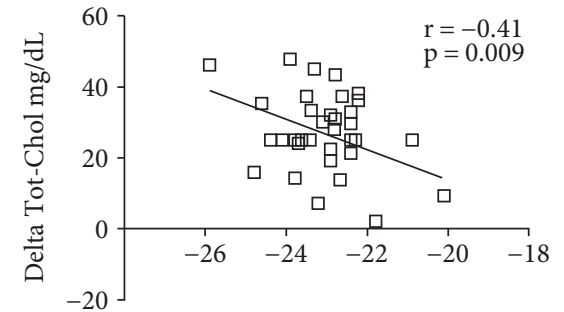

Delta CDAI
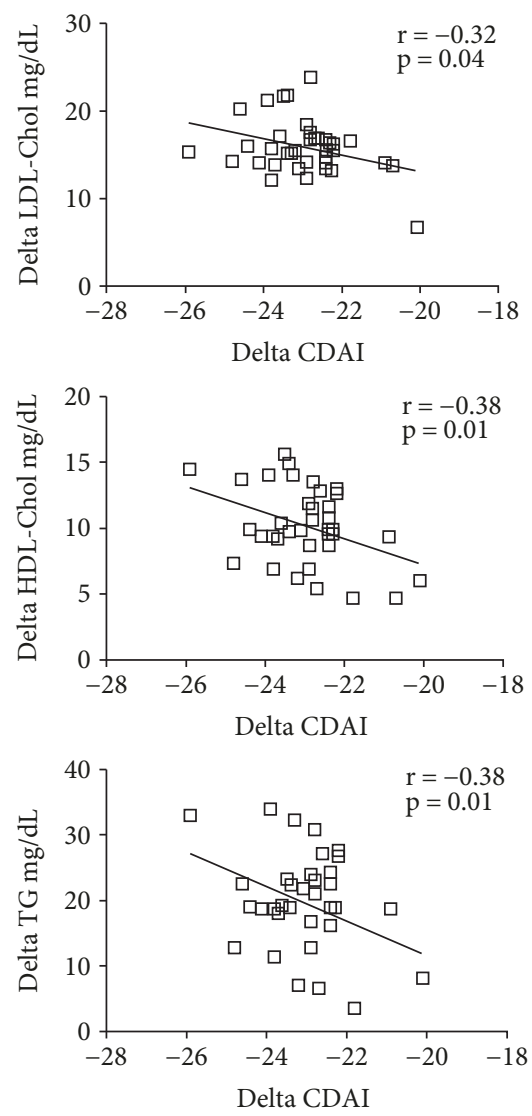

(b)

FIgure 3: Correlations between variations (delta) in total cholesterol (Tot-Chol), low-density lipoprotein (LDL) and high-density lipoprotein (HDL) cholesterol, and triglyceride (TG) plasmatic levels and variation (delta) of the DAS28 (a) and CDAI (b) after 24 weeks of tocilizumab treatment.

progressively return to a lipid metabolism regulated by the same factors of subjects without RA.

Our findings confirmed that TCZ may influence the cholesterol levels and the other lipid fractions with a comparable gain of the different lipoproteins, and we demonstrated therefore that the different lipid ratios remain unaltered. Accordingly, other authors have reported an increase, after 3 months, of all major lipoproteins during treatment with TCZ, more evident for the LDL-Chol, but with relative stability of both LDL-Chol/HDL-Chol and Tot-Chol/HDL-Chol ratios. In addition, we have demonstrated that AIP does not change during 12 months of TCZ treatment. These parameters are known to be more closely associated to the real $\mathrm{CV}$ risk due to lipids than the individual measures of lipid fractions, which may be mistaken by the effect of inflammation
$[35,36]$. To date, the AIP as a surrogate of small LDL particle size is considered a valid predictor of CV risk, especially in selected categories of patients, such as diabetics [26].

According to the inclusion criteria of our study, patients should not receive any lipid-lowering treatment, and considering the cholesterol levels achieved after 12 weeks of TCZ treatment, some patients would be eligible for such therapy. Indeed, the EULAR recommendations on CV risk management in inflammatory arthritis suggest starting statin [37], but in the case of TCZ treatment and its metabolic effects, this decision could be procrastinated after at least 6 months of drug use, also to avoid the potential muscle side effects of statin [38]. Moreover, our observation of lipid fraction increase associated with TCZ treatment should be considered "paradoxical" on CV risk, underlining once again the 
complex interaction between systemic inflammation, lipids, and $\mathrm{CV}$ risk in RA patients.

It has been demonstrated that disease activity indices were independently correlated to CV risk in RA patients, as well as a high atherogenic index at the baseline (pretreatment) may be independent of the inhibition of IL-6. Furthermore, poor control of disease activity in RA but not necessarily lipid changes, has been associated with occurrence of major adverse CV events in patients treated with TCZ [39].

Finally, the correlation of changes in disease activity and Tot-Chol, HDL, LDL, and TG levels demonstrates that the systemic inflammatory state has metabolic effects. The correlation especially between lipid fraction and CDAI, calculated without considering the CRP with hepatic secretion according to IL-6 circulating levels [40], suggests that disease activity may have a direct influence on lipid profile of RA patients.

The clear effect of these changes on the lipid-dependent $\mathrm{CV}$ risk should be demonstrated with long-term prospective studies without the interference of any other drugs on the lipid set.

\section{Conclusions}

Our study, with a 1-year follow-up, confirmed that TCZ may interfere with cholesterol levels; we demonstrated a more consistent effect during the first 6 months of treatment and subsequently a reverse trend to pretreatment levels.

We found a similar effect on all lipoprotein fractions; thus, no substantial differences were highlighted in the evaluated lipid ratios, Tot-Chol/HDL-Chol and LDL-Chol/ HDL-Chol, and the AIP, and the net effect on lipid-related $\mathrm{CV}$ risk would seem irrelevant.

Further studies are needed to confirm our findings and to elucidate the intriguing mechanisms linking inflammation and lipid metabolism.

\section{Data Availability}

The data about TCZ and MTX effects on lipid profile used to support the findings of this study are included within the article.

\section{Conflicts of Interest}

The authors declared they do not have any conflict of interest with respect to this manuscript.

\section{Authors' Contributions}

FC, GLa, and FI designed the study and wrote the final draft, and FC, MGA, AR, MF, CS, and GLo enrolled patients and are responsible for the data collection. FC, MF, and FI completed the statistical analysis. FC, MGA, AR, and MF performed the bibliographic research.

\section{References}

[1] S. E. Gabriel, C. S. Crowson, H. M. Kremers et al., "Survival in rheumatoid arthritis: a population-based analysis of trends over 40 years," Arthritis and Rheumatism, vol. 48, no. 1, pp. 54-58, 2003.

[2] M. A. Gonzalez-Gay, C. Gonzalez-Juanatey, and J. Martin, "Rheumatoid arthritis: a disease associated with accelerated atherogenesis," Seminars in Arthritis and Rheumatism, vol. 35, no. 1, pp. 8-17, 2005.

[3] M. Dougados, M. Soubrier, A. Antunez et al., "Prevalence of comorbidities in rheumatoid arthritis and evaluation of their monitoring: results of an international, cross-sectional study (COMORA)," Annals of the Rheumatic Diseases, vol. 73, no. 1, pp. 62-68, 2013.

[4] M. A. Gonzalez-Gay, C. Gonzalez-Juanatey, A. Pineiro, C. Garcia-Porrua, A. Testa, and J. Llorca, "High-grade Creactive protein elevation correlates with accelerated atherogenesis in patients with rheumatoid arthritis," The Journal of Rheumatology, vol. 32, no. 7, pp. 1219-1223, 2005.

[5] E. Choy, K. Ganeshalingam, A. G. Semb, Z. Szekanecz, and M. Nurmohamed, "Cardiovascular risk in rheumatoid arthritis: recent advances in the understanding of the pivotal role of inflammation, risk predictors and the impact of treatment," Rheumatology, vol. 53, no. 12, pp. 2143-2154, 2014.

[6] C. S. Crowson, S. Rollefstad, G. D. Kitas et al., "Challenges of developing a cardiovascular risk calculator for patients with rheumatoid arthritis," PLoS One, vol. 12, no. 3, article e0174656, 2017.

[7] P. Libby, "Role of inflammation in atherosclerosis associated with rheumatoid arthritis," The American Journal of Medicine, vol. 121, no. 10, Supplement 1, pp. S21-S31, 2008.

[8] Z. Szekanecz, G. Kerekes, H. der et al., "Accelerated atherosclerosis in rheumatoid arthritis," Annals of the New York Academy of Sciences, vol. 1108, no. 1, pp. 349-358, 2007.

[9] J. Rodríguez-Carrio, M. Alperi-López, P. López et al., "High triglycerides and low high-density lipoprotein cholesterol lipid profile in rheumatoid arthritis: a potential link among inflammation, oxidative status, and dysfunctional high-density lipoprotein," Journal of Clinical Lipidology, vol. 11, no. 4, pp. 1043-1054.e2, 2017.

[10] S. Ajeganova, M. L. E. Andersson, J. Frostegard, and I. Hafstrom, "Disease factors in early rheumatoid arthritis are associated with differential risks for cardiovascular events and mortality depending on age at onset: a 10-year observational cohort study," The Journal of Rheumatology, vol. 40, no. 12, pp. 1958-1966, 2013.

[11] E. Myasoedova, C. S. Crowson, H. M. Kremers et al., "Lipid paradox in rheumatoid arthritis: the impact of serum lipid measures and systemic inflammation on the risk of cardiovascular disease," Annals of the Rheumatic Diseases, vol. 70, no. 3, pp. 482-487, 2011.

[12] H. K. Choi, M. A. Hernan, J. D. Seeger, J. M. Robins, and F. Wolfe, "Methotrexate and mortality in patients with rheumatoid arthritis: a prospective study," Lancet, vol. 359, no. 9313, pp. 1173-1177, 2002.

[13] W. G. Dixon, K. D. Watson, M. Lunt et al., "Reduction in the incidence of myocardial infarction in patients with rheumatoid arthritis who respond to anti-tumor necrosis factor alpha therapy: results from the British Society for Rheumatology Biologics Register," Arthritis and Rheumatism, vol. 56, no. 9, pp. 2905-2912, 2007.

[14] A. A. Mangoni, A. Zinellu, S. Sotgia, C. Carru, M. Piga, and G. L. Erre, "Protective effects of methotrexate against proatherosclerotic cytokines: a review of the evidence," 
Mediators of Inflammation, vol. 2017, Article ID 9632846, 11 pages, 2017.

[15] D. T. Lim, A. C. Cannella, K. D. Michaud, and T. R. Mikuls, "Cardiovascular risk and the use of biologic agents in rheumatoid arthritis," Current Rheumatology Reports, vol. 16, no. 11, p. 459, 2014.

[16] A. C. Strang, R. J. Bisoendial, R. S. Kootte et al., "Pro-atherogenic lipid changes and decreased hepatic LDL receptor expression by tocilizumab in rheumatoid arthritis," Atherosclerosis, vol. 229, no. 1, pp. 174-181, 2013.

[17] A. Souto, E. Salgado, J. R. Maneiro, A. Mera, L. Carmona, and J. J. Gomez-Reino, "Lipid profile changes in patients with chronic inflammatory arthritis treated with biologic agents and tofacitinib in randomized clinical trials: a systematic review and meta-analysis," Arthritis \& Rhematology, vol. 67, no. 1, pp. 117-127, 2015.

[18] P. Welsh, K. Tuckwell, I. B. McInnes, and N. Sattar, "Effect of IL-6 receptor blockade on high-sensitivity troponin T and NTproBNP in rheumatoid arthritis," Atherosclerosis, vol. 254, pp. 167-171, 2016.

[19] K. Kume, K. Amano, S. Yamada, K. Hatta, H. Ohta, and N. Kuwaba, "Tocilizumab monotherapy reduces arterial stiffness as effectively as etanercept or adalimumab monotherapy in rheumatoid arthritis: an open-label randomized controlled trial," The Journal of Rheumatology, vol. 38, no. 10, pp. 2169-2171, 2011.

[20] C. Gabay, I. B. McInnes, A. Kavanaugh et al., "Comparison of lipid and lipid-associated cardiovascular risk marker changes after treatment with tocilizumab or adalimumab in patients with rheumatoid arthritis," Annals of the Rheumatic Diseases, vol. 75, no. 10, pp. 1806-1812, 2016.

[21] J. Robertson, D. Porter, N. Sattar et al., "Interleukin-6 blockade raises LDL via reduced catabolism rather than via increased synthesis: a cytokine-specific mechanism for cholesterol changes in rheumatoid arthritis," Annals of the Rheumatic Diseases, vol. 76, no. 11, pp. 1949-1952, 2017.

[22] D. Aletaha, T. Neogi, A. J. Silman et al., "2010 rheumatoid arthritis classification criteria: an American College of Rheumatology/European League Against Rheumatism collaborative initiative," Annals of the Rheumatic Diseases, vol. 69, no. 9, pp. 1580-1588, 2010.

[23] A. M. van Gestel, M. L. Prevoo, M. A. van 't Hof, M. H. van Rijswijk, L. B. van de Putte, and P. L. van Riel, "Development and validation of the European League Against Rheumatism response criteria for rheumatoid arthritis. Comparison with the preliminary American College of Rheumatology and the World Health Organization/International League Against Rheumatism criteria," Arthritis and Rheumatism, vol. 39, no. 1, pp. 34-40, 1996.

[24] I. Lemieux, B. Lamarche, C. Couillard et al., "Total cholesterol/ HDL cholesterol ratio vs LDL cholesterol/HDL cholesterol ratio as indices of ischemic heart disease risk in men: the Quebec Cardiovascular Study," Archives of Internal Medicine, vol. 161, no. 22, pp. 2685-2692, 2001.

[25] V. Bittner, "Non-high-density lipoprotein cholesterol: an alternate target for lipid-lowering therapy," Preventive Cardiology, vol. 7, no. 3, pp. 122-130, 2004.

[26] A. Onat, G. Can, H. Kaya, and G. Hergenc, “" Atherogenic index of plasma" ( $\log _{10}$ triglyceride/high-density lipoprotein-cholesterol) predicts high blood pressure, diabetes, and vascular events," Journal of Clinical Lipidology, vol. 4, no. 2, pp. 89-98, 2010.
[27] S. M. Grundy, J. I. Cleeman, C. N. Merz et al., "Implications of recent clinical trials for the National Cholesterol Education Program Adult Treatment Panel III guidelines," Journal of the American College of Cardiology, vol. 44, no. 3, pp. 720-732, 2004.

[28] Expert Panel on Detection, Evaluation, and Treatment of High Blood Cholesterol in Adults, "Executive summary of the third report of the National Cholesterol Education Program (NCEP) expert panel on detection, evaluation, and treatment of high blood cholesterol in adults (adult treatment panel III)," Journal of the American Medical Association, vol. 285, no. 19, pp. 2486-2497, 2001.

[29] W. B. Kannel, "Risk stratification of dyslipidemia: insights from the Framingham study," Current Medicinal Chemistry. Cardiovascular and Hematological Agents, vol. 3, no. 3, pp. 187-193, 2005.

[30] J. Robertson, M. J. Peters, I. B. McInnes, and N. Sattar, "Changes in lipid levels with inflammation and therapy in RA: a maturing paradigm," Nature Reviews Rheumatology, vol. 9, no. 9, pp. 513-523, 2013.

[31] M. Hashizume, H. Yoshida, N. Koike, M. Suzuki, and M. Mihara, "Overproduced interleukin 6 decreases blood lipid levels via upregulation of very-low-density lipoprotein receptor," Annals of the Rheumatic Diseases, vol. 69, no. 4, pp. 741-746, 2010.

[32] I. B. McInnes, L. Thompson, J. T. Giles et al., "Effect of interleukin-6 receptor blockade on surrogates of vascular risk in rheumatoid arthritis: MEASURE, a randomised, placebocontrolled study," Annals of the Rheumatic Diseases, vol. 74, no. 4, pp. 694-702, 2015.

[33] C. García-Gómez, M. A. Martín-Martínez, S. Castañeda et al., "Lipoprotein(a) concentrations in rheumatoid arthritis on biologic therapy: results from the CARdiovascular in rheuMAtology study project," Journal of Clinical Lipidology, vol. 11, no. 3, pp. 749-756.e3, 2017.

[34] J. L. Goldstein and M. S. Brown, “The LDL receptor," Arteriosclerosis, Thrombosis, and Vascular Biology, vol. 29, no. 4, pp. 431-438, 2009.

[35] S. S. Mahmood, D. Levy, R. S. Vasan, and T. J. Wang, "The Framingham Heart Study and the epidemiology of cardiovascular disease: a historical perspective," Lancet, vol. 383, no. 9921, pp. 999-1008, 2014.

[36] S. Natarajan, H. Glick, M. Criqui, D. Horowitz, S. R. Lipsitz, and B. Kinosian, "Cholesterol measures to identify and treat individuals at risk for coronary heart disease," American Journal of Preventive Medicine, vol. 25, no. 1, pp. 50-57, 2003.

[37] R. Agca, S. C. Heslinga, S. Rollefstad et al., "EULAR recommendations for cardiovascular disease risk management in patients with rheumatoid arthritis and other forms of inflammatory joint disorders: 2015/2016 update," Annals of the Rheumatic Diseases, vol. 76, no. 1, pp. 17-28, 2016.

[38] A. Tiwari, V. Bansal, A. Chugh, and K. Mookhtiar, "Statins and myotoxicity: a therapeutic limitation," Expert Opinion on Drug Safety, vol. 5, no. 5, pp. 651-666, 2006.

[39] V. U. Rao, A. Pavlov, M. Klearman et al., "An evaluation of risk factors for major adverse cardiovascular events during tocilizumab therapy," Arthritis \& Rhematology, vol. 67, no. 2, pp. 372-380, 2015.

[40] T. Tanaka, M. Narazaki, A. Ogata, and T. Kishimoto, "A new era for the treatment of inflammatory autoimmune diseases by interleukin- 6 blockade strategy," Seminars in Immunology, vol. 26, no. 1, pp. 88-96, 2014. 


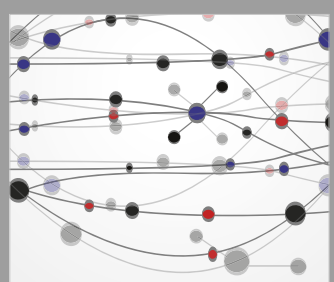

The Scientific World Journal
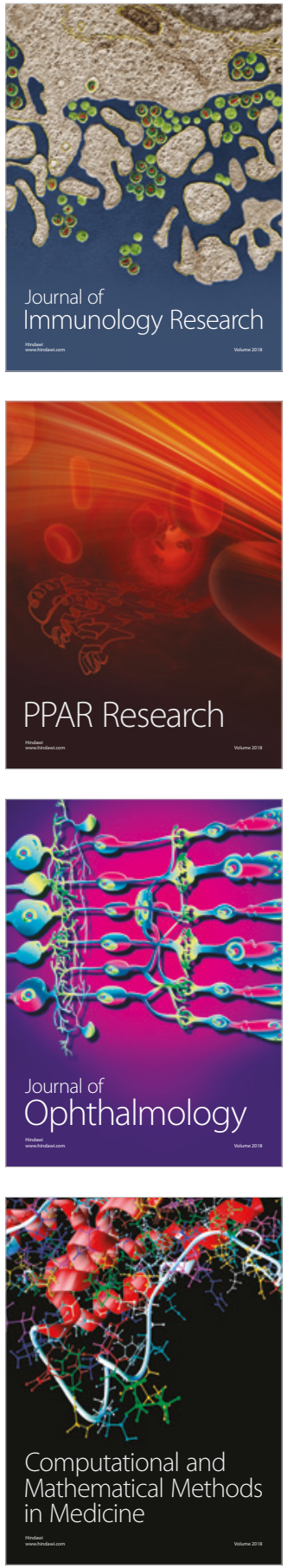

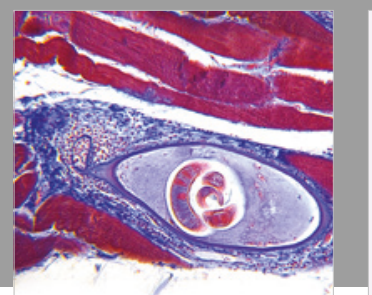

Gastroenterology Research and Practice

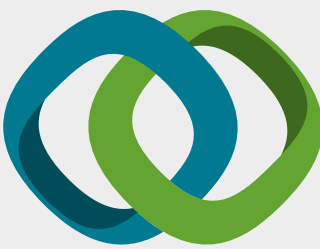

\section{Hindawi}

Submit your manuscripts at

www.hindawi.com
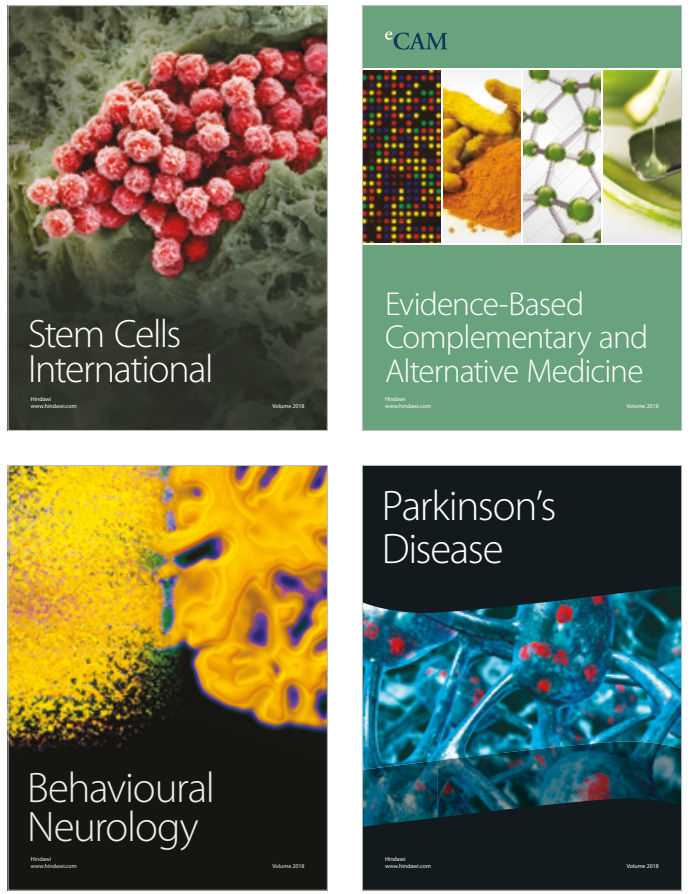

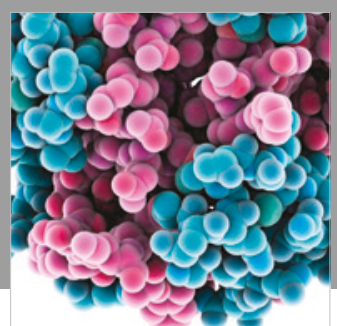

ournal of

Diabetes Research

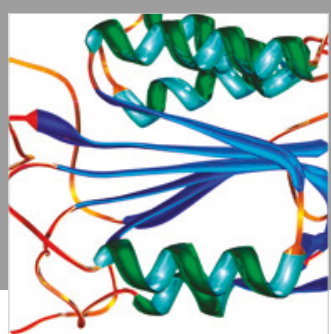

Disease Markers
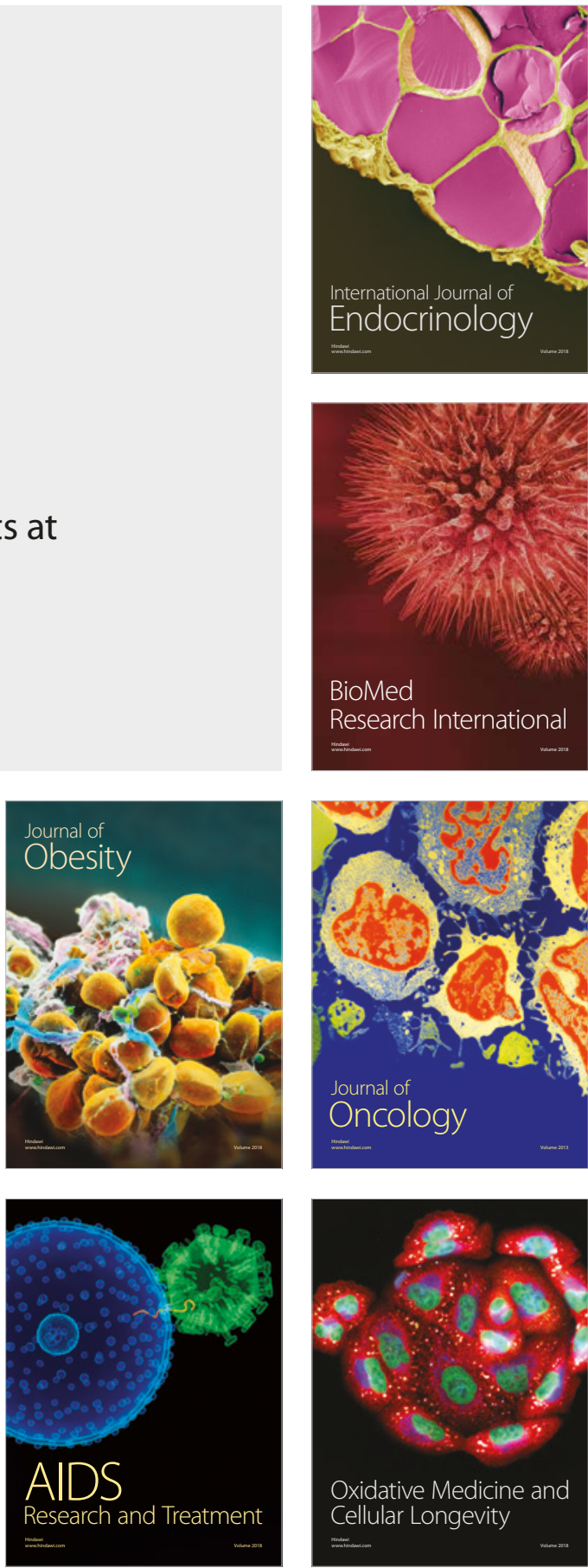